\title{
Aprovechamiento racional de los recursos naturales y culturales para el turismo comunitario, cantón Montalvo-Los Ríos
}

Rational use of natural and cultural resources for community tourism, canton Montalvo-Los Ríos.

Christian Rivera García. ${ }^{1}$, Maricela Izurieta Puente. ${ }^{2}, \&$ Arely Cuadro Saucedo. ${ }^{3}$

\begin{abstract}
.
DOI: https://doi.org/10.33262/cienciadigital.v3i3.619

Local development is based on tourism initiatives of rural sustainability. The territorial dynamics of the indigenous communities, atomize the multiple possibilities of forming clusters of support to entrepreneurship initiatives that are cordoned off to previously established plans in different branches of local, regional, national and international organization. Intensifying the rescue and creation of recreational and leisure activities is the challenge of the new page of the multi-ethnic and biodiverse Ecuador. These efforts motivate the coupling of tourism initiatives such as integrating customs, traditions and ancestral knowledge to enhance community tourism, which agglomerate ideas of young entrepreneurs who multiply their ideas seeking to innovate in increasingly competitive markets. These ventures have to face much more differentiating challenges, human losses particularly due to hunger and diseases, this has compromised planning to provide relief and human control over the shell of impunity in sectors that really need transformation support and support for initiatives of great importance It must be remembered that tourism is not the solution to certain population setbacks, but it is a great help to address possible solutions according to the social interest of tourism regeneration, as an advantage of natural and cultural conservation with maximum opportunities for local development. Tourism demand responds to globalizing trends that diagnose, design, execute and value productive
\end{abstract}

\footnotetext{
${ }^{1}$ Universidad Técnica de Babahoyo, Ecuador, crivera@utb.edu.ec

${ }^{2}$ Universidad Técnica de Babahoyo, Ecuador, eizurieta@utb.edu.ec

${ }^{3}$ Universidad Técnica de Babahoyo, Ecuador, arelycuadro@gmail.com
} 
initiatives of great importance, providing reorganize activities for the enjoyment and distraction of travelers.

Keywords: Local Development, Resources, Sustainable Development, Community Tourism, Entrepreneurship

\section{Resumen.}

El desarrollo local se sustenta en iniciativas turísticas de sostenibilidad rural. La dinámica territorial de las comunidades indígenas, atomizan las múltiples posibilidades de formar clústeres de apoyo a iniciativas de emprendimiento que están acordonadas a planificaciones previamente establecidas en diferentes ramales de organización local, regional, nacional e internacional. Intensificar el rescate y creación de actividades recreativas y de descanso es el reto de la nueva página del Ecuador multiétnico y biodiverso. Estos esfuerzos motivan el acoplamiento de iniciativas turísticas como integrar costumbres, tradiciones y saberes ancestrales para potenciar el turismo comunitario, que aglomeran ideas de jóvenes emprendedores que multiplican sus ideas buscando innovar en mercados cada vez más competitivos. Estos emprendimientos tienen que enfrentar retos mucho más diferenciadores, pérdidas humanas particularmente por el hambre y enfermedades, esto ha comprometido realizar planificaciones que brinden desahogo de estima y control humano sobre la coraza de impunidad en sectores realmente que necesitan ayuda de transformación y apoyo a iniciativas de gran importancia. Se tiene que recordar que el turismo no es la solución a ciertos contratiempos poblacionales, pero si es una gran ayuda para direccionar posibles soluciones de acuerdo a interés social de regeneración turística en ventaja de conservación natural y cultural con máximas oportunidades de desarrollo local. La demanda turística responde a tendencias globalizadoras que diagnóstica, diseña, ejecuta y valora iniciativas productivas de gran calado, proporcionando reorganizar actividades para el disfrute y distracción de los viajeros.

Palabras Calves: Desarrollo Local, Desarrollo Sostenible, Turismo Comunitario, Emprendimientos

\section{Introducción.}

El Turismo Comunitario establece en la actualidad una oportunidad y una estrategia de desarrollo local para las comunidades rurales que permite aprovechar y conservar la potencialidad de los recursos naturales y culturales de manera sostenible.

Desde la década de los setenta, organismos internacionales han promovido al turismo como fórmula del desarrollo en los países de Europa, en España el turismo comunitario se centra en mejorar su situación económica y conservar los recursos naturales de un territorio, el turismo comunitario ha implementado una diversificación en la oferta turística por las comunidades, mientras que la potencialidad de las comunas es diferente en preservar el 
paisaje, el patrimonio y la cultura. En Portugal el turismo comunitario surge como una alternativa económica de las comunidades rurales como en Azores generan ingresos complementarios a las actividades económicas diarias, defendiendo sus costumbres y creencias, en la actualidad han sumado la capacidad de alojamiento rural, generando mayor oferta turística. Maldonado, C. (2005)

Australia y el Caribe, se destaca su importancia como fuente de riqueza de las economías nacionales, lo que ha sido fundamental para que los gobiernos adopten y promuevan el desarrollo turístico como una forma de generar empleos, inversiones y divisas. En Australia el turismo comunitario es una alternativa diferente que beneficia a una población local principalmente a los pueblos indígenas y campesinos de zonas rurales. En el Caribe el turismo comunitario se refiere a diversificar e incrementar la economía rural, sin embargo las actividades fundamentales de los territorios como son la agricultura, la pesca, la artesanía y la gestión forestal, cobrando un importante valor puesto que en la región hay un número grande de comunidades que han creado una oferta turística propia, donde las organizaciones comunitarias deciden como realizar las actividades turísticas y como se deben distribuir lo que proviene de esta actividad. Castillo, A. (1999)

El Turismo Comunitario en América Latina es un fenómeno que data de las dos últimas décadas y surge en un contexto de grandes cambios económicos, sociales y políticos: con un relativo incremento en zonas de excepcional belleza paisajística, dotadas de atractivos culturales y vida silvestre, ya sea en territorios comunales o en áreas públicas aledañas. En el Salvador el turismo comunitario lo consideran como una forma de desarrollar el turismo en espacios rurales donde cooperativas, familias, pueblos indígenas y otras organizaciones comunales realizan actividades turísticas cuidando los recursos que se encuentran en su territorio. Azevedo, L. (2007)

Esta actividad se va posicionando como un nuevo elemento en la oferta turística, se lo considera, como uno de los múltiples productos turísticos que se ofrecen en el mercado. Las comunidades en el área rural ofrecen: vacaciones con relación naturaleza y cultura, observación de flora y fauna, convivencia con la población, aprendizaje de sus tradiciones, actividades deportivas como el senderismo, que se realizan en la mayoría de países donde se oferta esta clase de turismo. En Sudamérica cada vez es mayor la cantidad de turistas que practican otras alternativas de esparcimiento y recreación como el contacto con la naturaleza, deportes de aventura y vivencias con la comunidad. Organización Mundial del Turismo (OMT) (2012b).

Esta preocupación se visibiliza en los contrastes de la población en sus características antropológicas, la producción territorial y su identidad cultural, anclando el desarrollo local de interacciones a las bases amplias, participación comunitaria, desarrollo holístico y la desconcentración de la empresa conjunta que sutilizan el uso racional de los recursos naturales y culturales como materia prima, en aumento de la contaminación y sobre 
explotación de la tierra, ausentando su valor irremediable e impostergable sobre los beneficios del turismo comunitario.

Los elementos sobresalientes de este tipo de turismo son los recursos culturales y los recursos naturales. El clima, condiciones físicas, geológicas, hídricas, morfológicas, relieve, flora, fauna entre otras, influyen en el desempeño histórico y económico de la población, integrado todo en la actividad turística, que en ningún lugar es hegemónica o aleatoria, sino puntual, geográfica y sostenible.

El Desarrollo Sostenible busca satisfacer las necesidades de generaciones actuales sin comprometer generaciones futuras para satisfacer las necesidades, permitiendo a las personas en el presente y en el futuro alcanzar un nivel de desarrollo social, económico, el logro humano y cultural para que con el tiempo se impulse el uso razonable de los recursos ambientales preservando la tierra, las especies y los hábitats naturales para no agotar los recursos en el futuro, este requiere el conocimiento de que los recursos son finitos teniendo como objetivo el desarrollo económico, el desarrollo social y la conservación ambiental. El desarrollo sostenible tiene cuatro componentes con los que se caracteriza su definición.

La sostenibilidad ambiental, mantiene los servicios ambientales viables capaces de adaptarse a cambios que mantengan su variedad biológica. La capacidad del medioambiente tiene que mantener sus condiciones ambientales y la belleza del entorno.

La sostenibilidad cultural es la forma que constituye la gestión de las relaciones con las personas ya sea corto o largo plazo creando un mundo más sostenible para los niveles sociales garantizando la participación local.

Según la Organización Mundial del Turismo (OMT,2009) El turismo comunitario se asoma como una alternativa para salvar las disparidades sociales y económicos que adolecen las comunidades rurales, además de una vía que pueden utilizar las comunidades que buscan generar otros ingresos dentro de sus actividades económicas cotidianas, utilizando los recursos culturales, naturales y locales en una región en particular constituyendo una de las principales industrias generadoras de divisas a nivel mundial con tendencia al crecimiento y especialización.

Según los Objetivos del Desarrollo Sostenible (ODS, 2016) Fortalecerán las metas alcanzadas por los Objetivos del Desarrollo del Milenio (ODM) y dirigir los esfuerzos gubernamentales hacia otras áreas para el desarrollo sostenible de todos los países del mundo. Se espera que este programa genere debate en torno a muchas cuestiones de esfuerzos importantes para la erradicación de la pobreza y el hambre, la mejora de los servicios de salud y educación, nuevos modelos de desarrollo para ciudades más sostenibles, inteligentes y 
seguras, así como políticas coherentes para enfrentar el cambio climático, entre otros importantes temas.

El Objetivo 1 del Desarrollo Sostenible describe como la pobreza ha tomado posesión de los países menos desarrollados generando déficit, en la China y la India el crecimiento económico acelerado ayudado a salir a millones de persona de pobreza pero el beneficio ha sido disparejo, el compromiso de los ODS es la erradicación de la pobreza en todas las formas garantizando al sector vulnerable medios suficientes como la alimentación, la educación, y los servicios básicos.

El Objetivo 2 del Desarrollo Sostenible propone terminar con el hambre asegurando a los pobres una alimentación nutritiva poniendo fin a la mal nutrición duplicando la producción agrícola asegurando la sostenibilidad de los sistemas de producción de alimentos manteniendo la diversidad genética de la semilla, apoyando a la agricultura silvicultura y ganadería aparte de incrementar los ingresos económicos, genera suministros para una alimentación, conservando al medio ambiente.

El Objetivo 3 del Desarrollo Sostenible promueva la reducción de la tasa de mortalidad poniendo fin a las muertes evitables a los recién nacidos erradicando enfermedades catastróficas fortaleciendo el compromiso con la Organización Mundial de la Salud OMS, con los ODS se garantiza una vida saludable apoyando las actividades de vacunación y medicamentos a los actores más vulnerables como a la salud infantil y la salud materna.

El Objetivo 4 del Desarrollo Sostenible promete velar por los niños y niñas terminen el ciclo de enseñanza primaria, servicios de atención y desarrollo asegurando la igual de genero aumentando la sustancialmente el número de becas, garantizando la educación de elite para todos los sectores creando instalaciones adecuadas para las necesidades de los niños y los discapacitados dándoles una educación digna y decente.

El Objetivo 10 del Desarrollo Sostenible propone lograr mantener los ingresos del $40 \%$ de la población más pobre promoviendo la inclusión social, económica y política, garantizando la igualdad de oportunidades alentando al sistema financiero para el desarrollo corriente, implementando pariedades en los servicios sanitarios, educación y los bienes productivos.

El Objetivo 11 del Desarrollo Sostenible pretende el acceso de viviendas servicio de transporte público a todas las personas, reducir el número de muertes por desastres naturales y promover las políticas urbanas locales y nacionales sostenibles, para mejorar la planificación del diseño del espacio optimizando la diversidad, abogando por un financiamiento justo.

El Objetivo 12 del Desarrollo Sostenible proyecta la gestión sostenible y el uso suficiente de los recursos naturales reduciendo el desperdicio de alimentos, reduciendo la producción de desechos mediante políticas de prevención, generando conciencia para mejorar la calidad de 
vida de los individuos concientizando el responsable consumo de los recursos, infraestructura sostenible, facilitando el acceso a los recursos básicos, creando conciencia en la cadena de suministros desde el agricultor hasta el consumidor.

El Objetivo 15 del Desarrollo Sostenible promete velar por la conservación y restablecer el ecosistema terrestre gestionando la sostenibilidad de todos los bosques adoptando medidas para reducir la degradación de los hábitats, reflexionando sobre los recursos naturales conservando el ecosistema, erradicando la caza indiscriminada, el tráfico de especies protegidas, las especies en peligro de extinción y la diversidad biológica.

El Objetivo 17 del Desarrollo Sostenible ambiciona fortalecer la movilización de los recursos internos velando que los países cumplan a cabalidad sus compromisos ayudando a la sostenibilidad, aumentando el apoyo internacional a la ejecución de programas de planes nacionales, los objetivos sirven para impulsar al trabajo conjunto formando alianzas con el gobierno, el sector privado y el civil para de esa manera fortalecer nexos mundiales de desarrollo sostenible y el intercambio de conocimientos aprovechando la experiencia y las estrategias de los recursos asociados.

https://www.un.org/sustainabledevelopment/es/objetivos-de-desarrollo-sostenible/

La Alianza Centroamericana Para el Desarrollo Sostenible (ALIDES, 2017) tiene como una iniciativa de políticas, programas y acciones a corto, mediano y largo plazo que delinea un cambio de esquema de desarrollo, de nuestras actitudes individuales y colectivas, de las políticas y acciones locales, nacionales y regionales hacia la sostenibilidad política, económica, social, cultural y ambiental de las sociedades, esta es una estrategia regional de coordinación y concertación de intereses, iniciativas de desarrollo, responsabilidades y armonización de derechos. Su implementación se apoya en la institucionalidad y no sustituye los mecanismos o instrumentos de integración regional existentes, sino que los complementa, apoya y fortalece, intrarregional y extra regionalmente, en especial en su proceso de convertir el desarrollo sostenible en la estrategia y política central de los estados y de la región en su conjunto. Mediante la alianza se reiteran y amplían los compromisos ya contraídos por los estados para el nuevo proceso de desarrollo sostenible en el istmo. En este esfuerzo y compromiso de desarrollo sostenible, propio de la comunidad centroamericana, asumimos las responsabilidades para un mejor aprovechamiento y manejo eficiente de los recursos de nuestra región. En este sentido, consideramos que la comunidad internacional puede y debe contribuir al desarrollo sostenible centroamericano, por medio de un cambio de sus propias actitudes, políticas y acciones hacia esta región, lo que redefinirá integralmente las relaciones entre la comunidad internacional.

Según el Plan Nacional del Buen Vivir (PNBV) busca realizar el cambio en la economía mejorando la calidad de vida promoviendo la diversidad cultural. Generar riqueza y elevar en forma general el nivel de vida de nuestra población, que el país debe gestionar sus recursos 
estratégicos en el marco de una inserción internacional, que permite el incremento generalizado del bienestar para sus habitantes, de tal punto que el estado ecuatoriano a visto como una estrategia para la integración cultural como herramienta esencial en el desarrollo de nuestro país.

El Objetivo 2 del Plan Nacional del Buen Vivir describe que se debe reconocer los derechos de los ciudadanos con igualdad, consolidando políticas que eviten exclusión fortaleciendo la convivencia social, avanzando hacia la plena diversidad para lograr una vida digna con acceso a la salud, protección social, protección especial y atención especializada.

El Objetivo 3 del Plan Nacional del Buen Vivir dice que es una alternativa para fortalecer la calidad de vida de la ciudadanía en base de las necesidades que la sociedad tenga y muestre, buscando condiciones para fortalecer la capacidad pública manteniendo el fortalecimiento de políticas intersectoriales consolidando el sistema nacional de inclusión y equidad social.

El Objetivo 5 del Plan Nacional del Buen Vivir explica que el estado promueve las políticas igualitarias para asegurar las condiciones de la diversidad, construyendo la identidad cultural circulando los elementos simbólicos que representan la cultura tangible e intangible, el patrimonio y las memorias colectivas e individuales.

El Objetivo 7 del Plan Nacional del Buen Vivir propone el derecho ciudadano a vivir en un ambiente sano, libre de contaminación y sustentable, y la garantía de los derechos de la naturaleza, a través de una planificación integral que conserve los hábitats, gestione de manera eficiente los recursos, repare de manera integral e instaure sistemas de vida en una armonía real con la naturaleza.

El Objetivo 8 del Plan Nacional del Buen Vivir dice que las nuevas concepciones permitirán que la inclusión económica y social concrete la trasformación del modo de la producción, fortaleciendo las finanzas públicas, la justicia, la regulación del sistema económico y la igualdad con las condiciones laborales.

El Plan Territorial de la Zonal 5 la actividad turística genera fuentes de trabajo directos e indirectos en las áreas de alojamiento y servicios de alimentación, de acuerdo con los resultados nacionales de empleo, desempleo y subempleo en el Ecuador INEC 2015.Es esta zona la actividad está en buena posición por la llegada de turistas y los servicios que gozan en esta lugar.

En el Plan de Ordenamiento Territorial de la Provincia de Los Ríos, muestra que el turismo en nuestra provincia es un sector que aún no se ha desarrollado en su totalidad por lo que sería una oportunidad para los diferentes sectores de nuestro territorio en las que podemos mencionar a Montalvo, Vinces, Babahoyo, Baba, Valencia, Buena Fe y Quevedo, en los que encontramos una diversidad de alternativas como turismo de aventura, gastronómico, 
agroturismo, ecoturismo, religioso-cultural impulsando nuevas alianzas y estrategias; con el objetivo de implementar modelos innovadores de proceso local tales como incubadoras de empresas, industrias, parques tecnológicos; identificando organizaciones e instituciones como universidades, colegios, cámaras de comercio, industrias, gremios productivos, que promuevan el bien social para emprender programas, trabajos y actividades organizativasparticipativas con enfoque de género que permitan mejorar la capacidad de gestión y administración de los recursos tanto productivos, sociales y naturales.

En Ecuador el turismo comunitario es una alternativa, porque se lo considera un país con gran biodiversidad, por sus cadenas montañosas como la cordillera de los Andes, por la cadena volcánica, las corrientes marinas en el Ecuador varían según sus movimientos, la corriente del Niño, se caracteriza por ser de aguas cálidas provocando el aumento de la temperatura superficial del mar, la corriente de Humboldt es compleja por tener distintos componentes es más cálida y salina, los vientos Alisios son los que soplan los trópicos hacia el Ecuador como también los vientos Continentales son periódicos y estacionales, vierten su dirección con el paso de los días y noches con la sucesión de las estaciones.

Características geológicas y marinas donde conviven sociedades, culturas, grupos étnicos, pueblos y distintas nacionalidades, con lenguas, tradiciones históricas, cosmovisiones, formas de organización socio-políticas e identidades propias y diferenciadas, que hacen de este, un país multicolor, diverso, de múltiples rostros, donde se destaca por la solidaridad y generosidad de su gente, su identidad es el sello de la personalidad que los ecuatorianos tenemos por ser nuestra historia de vida que se relaciona con la época y el lugar en donde vivimos siendo fieles a nuestras creencias y costumbres que son arraigadas desde nuestros aborígenes que se vinculan con la naturaleza y las leyes del universo. Rivera C. et al. (2016)

Estos productos turísticos alternativos aparecen en diferentes comunidades donde se trata de establecer amistades diferentes, creando relaciones más cercanas con la población, esto permite observar las características de las multiculturalidades y las posibilidades que presta el país al turismo en sus diferentes manifestaciones. La iniciativa de turismo comunitario en las comunidades es incentivar al aprovechamiento de los diferentes beneficios que brinda este tipo de turismo. La región Litoral del Ecuador, ofrece varios lugares para visitar y convivir con las culturas, permitiendo experimentar una forma diferente, con la variedad de elementos de la naturaleza y la convivencia comunitaria. La forma de vida de sus habitantes muestra su historia, cultura, tradiciones y saberes ancestrales, que representa el nexo con el medio cultural exógeno, en perspectiva de la corresponsabilidad en el uso racional de los recursos naturales y culturales. Gallichio, E. (2004)

La provincia de Los Ríos se la conoce por sus innumerables elementos naturales de excepcional belleza, clima agradable, majestuosos ríos, cascadas, balnearios de agua dulce incomparables, festividades ancestrales en diferentes parroquias y comunidades que se destacan de acuerdo a sus fechas, su folclore, artesanías, complementadas con la 
infraestructura hotelera que ofrece un ambiente de paz y seguridad, varias opciones para el esparcimiento, descanso, enriquecimiento cultural y una reconocida hospitalidad.

El cantón Montalvo está situado a $32 \mathrm{~km}$ de Babahoyo, capital provincial, tierra mágica de aguas cristalinas, el santuario de la Virgen de los Remedios, la gastronomía típica y sus balnearios, que destacan al lugar. Esta zona estuvo habitada por grupos aborígenes, pertenecientes a los Babahuyus asentados a partir de las estribaciones de la cordillera occidental hacia las partes llanas. La producción agrícola y ganadera ennoblece el trabajo de las comunidades aledañas al cantón como, la comunidad de San Antonio, el nombre que lleva la comuna se originó a la llegada del párroco José María, a principios del siglo XIX, sostenía que esta devoción cristiana se debía a una herencia de los ancestros indígenas de los pobladores de la región tenían la costumbre de poner el nombre de santos a los recintos por esa razón proviene el nombre de San Antonio, también se dice que el nombre viene porque unos agricultores vivenciaron la aparición de San Antonio encima de una roca donde construyeron una capilla en nombre del Santo naciendo de ahí el nombre de la comunidad de San Antonio. Territorio rico en recursos naturales, recursos hídricos, como ríos, cascadas y diferentes manifestaciones culturales como: costumbres y juegos tradicionales, danzas folclóricas y su exquisita gastronomía, que enriquecen su patrimonio e identidad cultural. Según datos del INEC en el último censo que se realizó en el 2010 la población fue de 900 habitantes; el 32\% mujeres, el $52 \%$ hombres y el 14\% niños. Esta comunidad cuenta con todos los servicios básicos, recolector de basura y transporte público. Sin embargo, en la actualidad ninguna empresa proveedora de internet o servicio telefónico tienen cobertura en el lugar. Las viviendas mayoritariamente son financiadas por el Ministerio de Vivienda (MIDUVI). Es una comunidad de escasos recursos por su forma de vida que se caracteriza por el limitado acceso a los servicios tecnológicos, factores importantes que permiten impulsar el objetivo de establecer la implementación cultural, y alcanzar el mejoramiento de la potencialidad turística de sus habitantes para desarrollar un turismo sustentable en territorio.

Esta zona es de clima cálido-seco, con frecuentes precipitaciones lluviosas, especialmente en las épocas invernales lo que ha favorecido notablemente a los campos agrícolas y ganaderos. Su altitud es de 12 metros sobre el nivel del mar (m.s.n.m.) En cuanto a la orografía de esta comuna, podemos mencionar que se encuentra ubicada en un relieve regular, está limitada en la parte oriental por cordones montañosos, los principales ríos que se encuentran en este lugar encontramos a los ríos Las Piedras, y el Paraíso, cada uno constituye un sistema hidrográfico alimentado por pequeños esteros. La flora responde a varias especies de plantas comercializadoras como el cacao, maíz, banano, chontilla y árboles madereros productos que permiten la subsistencia de la población.

San Antonio es una comuna que presenta dificultades de accesibilidad, se ha detectado que gran parte de sus pobladores se dedican a actividades más lucrativas como: agricultura, ganadería, comercio entre otros, esta comunidad tiene gran potencialidad turística que debe 
ser aprovechada pero por el desinterés hacia las costumbres locales, y la inadecuada promoción de la superestructura turística complementaria, hacen que los miembros de la comuna no vislumbren la importancia al desarrollo del turístico comunitario.

\section{Metodología}

Tabla 1. Inquietudes territoriales como indicadores de desarrollo local turístico

\begin{tabular}{|l|c|l|}
\hline \multicolumn{1}{|c|}{$\begin{array}{c}\text { Aspectos conceptuales } \\
\text { importantes para la población }\end{array}$} & $\begin{array}{c}\text { Porcentaje de } \\
\text { aceptación } \\
\text { actitudinal }\end{array}$ & $\begin{array}{l}\text { Acciones procedimentales } \\
\text { en beneficio del } \\
\text { desarrollo territorial }\end{array}$ \\
\hline $\begin{array}{l}\text { La comunidad involucran la } \\
\text { práctica de costumbres propias del } \\
\text { sector con los visitantes. }\end{array}$ & $100 \%$ & $\begin{array}{l}\text { Ri } \\
\text { tradicionales. } \\
\text { Impulsar el reconocimiento } \\
\text { de las fiestas religiosas } \\
\text { Realizar ferias } \\
\text { gastronómicas }\end{array}$ \\
\hline $\begin{array}{l}\text { La comunidad considera, que la } \\
\text { asociatividad revaloriza las } \\
\text { costumbres locales }\end{array}$ & $\mathrm{Si}$ & $\begin{array}{l}\text { Formar parte de la } \\
\text { Federación Plurinacional } \\
\text { de Turismo Comunitario } \\
\text { del Ecuador (FEPCE) }\end{array}$ \\
\hline
\end{tabular}

Fuente: Elaboración propia de los autores

El método etnográfico y de observación permiten sistematizar expresiones sociales particulares que emergen por experiencias relatos, conversaciones que transcurren en la vida diaria de un individuo o en su conglomerado social, estas actividades son organizadas a través de técnicas de recolección de información como la encuesta y entrevista, cediendo a saberes ancestrales sobre un entorno en particular. Esta información imbrica sensaciones, emociones, hechos, valores o conductas expresadas por la población local. Esta investigación social se basó en parte, en información documental mundial y regional, para levantar el marco teórico sobre experiencias culturales y las necesidades turísticas básicas comunitarias, se elaboró diagnósticos participativos sobre las potencialidades poblacionales evidenciados la alegría de danzas folclóricas, juegos tradicionales, fiestas religiosas y los diferentes sabores gustativos.

\section{Resultados y Discusión}

La tabla anterior muestra evidencias sobre el comportamiento de la población frente a desafíos regionales, nacionales y mundiales, la idiosincrasia de nuestros pueblos es diluyente frente a las necesidades globales que exigen nuevas intervenciones comunitarias en integración de saberes ancestrales como carta de presentación turística frente al mundo cambiante, estos procesos turísticos como la asociatividad y la convivencia comunitaria, impulsan a conferir a las organizaciones plenamente reconocidas, iniciativas para 
implementar emprendimientos turísticos basado en el turismo comunitario que impera la realización de actividades comunes en mutuo aprovechamiento racional de recursos naturales y culturales prevaleciendo lo local en convivencia de acciones diarias compartidas con la legión de informales visitantes, temperando el accionar con manifestaciones lúdicas en su forma figurativa, fonética y simbólica. En la asociatividad, como norma de acción organizativa, prevalecen los intereses de la población en su conjunto y en la convivencia comunitaria como manifestación operativa prevalecen los intereses de los visitantes.

\section{Conclusiones.}

- La participación de la población en el trabajo comunitario es fundamental, la vinculación hacia el desarrollo sostenible, integra ámbitos de carácter grupal demostrado esto en la creación de redes participativas inclusivas, innovadoras y creativas líneas enmarcadas en la planificación nacional de formación y desarrollo de capacidades comunitarias mediante capacitaciones, talleres, asesoramiento técnico en busca de nuevos proyectos de interés mancomunado y asociativo con propuestas de gran valía sin interrumpir procesos empezados con anterioridad en aprovechamiento de los elementos endógenos y exógenos de la población rural.

- Construir comunidades competentes que refuercen sus indicadores de desarrollo con iniciativas y emprendimientos proyectistas es la misión del ministerio de turismo, la cámara provincial de turismo, la academia, entre otras instituciones que requieren diseñar en conjunto iniciativas de intervención comunitaria con polos opuestos de concentración de actividades alineadas en el Plan Nacional toda una vida.

\section{Referencias bibliográficas.}

Rivera C., Velasteguí E. \&, Vázquez Y. (2018). La sostenibilidad del turismo comunitario, desde una óptica milenaria. Revista electrónica Ciencia Digital 2(1), 405-419. Recuperado desdehttp://www.cienciadigital.org/revistascienciadigital2/index.php/

CienciaDigital/article/view/28/28

Alfonso, M. J. P. (2003). El patrimonio cultural como opción turística. Horizontes antropológicos, 9(20), 97-115.

Azevedo Luíndia, Luisa (2007). Ecoturismo de pueblos indígenas: propuestas sostenibles, La Paz, Bolivia: Fondo para el Desarrollo de los Pueblos Indígenas de América Latina y el Caribe.

Castillo, A. (1999). Estado del arte en la enseñanza del emprendimiento. Emprendedores como creadores de riqueza y desarrollo regional. 
Castillo, P. V., Venegas, C. B., Leiva, Y. F., Bennett, S. O., Ortiz, E. P., \& de Souza Neto, B. (2016). Una innovación pedagógica para la formación de universitarios emprendedores. Revista da FAE, 11(2).

Rivera García, C. Oviedo Rodríguez, M. Galarza Bravo F. (Abril 2016) Visión y Planeación Turística; Responsabilidad 100\% Humana (Pag. 60-70, 93,148).

Cohen, E. (2005). Principales tendencias en el turismo contemporáneo. Política y sociedad, 42(1), 11-24.

Gallichio, E. (2004). El desarrollo local:¿ cómo combinar gobernabilidad, desarrollo económico y capital social en el territorio?. Cuadernos del $\mathrm{CLAEH}, 27(89)$, 55-68.)

Gil, A. M. L. (2003). La evaluación del medio para la práctica de actividades turísticodeportivas en la naturaleza. Cuadernos de turismo, (12), 131-150.

(Gudynas, E. (2011). Buen Vivir: Germinando alternativas al desarrollo. América Latina en movimiento, 462, 1-20.)

Guerrero, Patricio. "Antecedentes". Notas para una guía teórica y metodológica sobre cultura, identidad, interculturalidad, patrimonio y turismo comunitario. 2008.

Mldonado, C. (2005). Pautas metodológicas para el análisis de experiencias de turismo comunitario. Organización Internacional del Trabajo, Ginebra, Suiza.

Organización Mundial del Turismo (OMT) (2012a). Barómetro del Turismo Mundial, volumen 10, julio de 2012.

Organización Mundial del Turismo (OMT) (2012b). Panorama OMT del turismo internacional. Organización Mundial del Turismo UNWTO, edición 2012.

Ruiz, E., Hernández, M., Coca, A., Cantero, P., \& Campo, A. D. (2008). Turismo comunitario en Ecuador. Comprendiendo el community-based tourism desde la comunidad. Pasos. Revista de turismo y patrimonio cultural, 6(3).

Salgado, J. R. (2003). Turismo Religioso: Alternativa de apoyo a la preservación del patrimonio y desarrollo. Revista Bibliográfica de Geografía y Ciencias Sociales, 6(316).

Sánchez, A. G., \& García, F. J. A. (2003). EL TURISMO CULTURALY EL DE SOLY PLAYA:¿̇ SUSTITUTIVOS O COMPLEMENTARIOS?. Cuadernos de turismo, (11), 97-106.

Sancho, A., \& Buhalis, D. (1998). Introducción al turismo. Madrid: Organización Mundial del Turismo. 


\section{PARA CITAR EL ARTÍCULO INDEXADO.}

Rivera García, C., Izurieta Puente, M., \& Cuadro Saucedo, A. (2019). Aprovechamiento racional de los recursos naturales y culturales para el turismo comunitario, cantón Montalvo-Los Ríos. Ciencia Digital, 3(3), 129-141. https://doi.org/10.33262/cienciadigital.v3i3.619

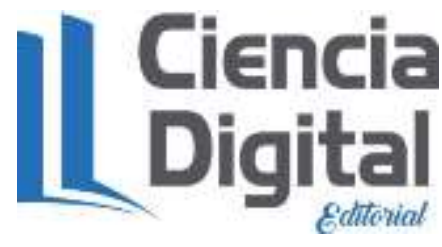

El artículo que se publica es de exclusiva responsabilidad de los autores y no necesariamente reflejan el pensamiento de la Revista Ciencia Digital.

El artículo queda en propiedad de la revista y, por tanto, su publicación parcial y/o total en otro medio tiene que ser autorizado por el director de la Revista Ciencia Digital.
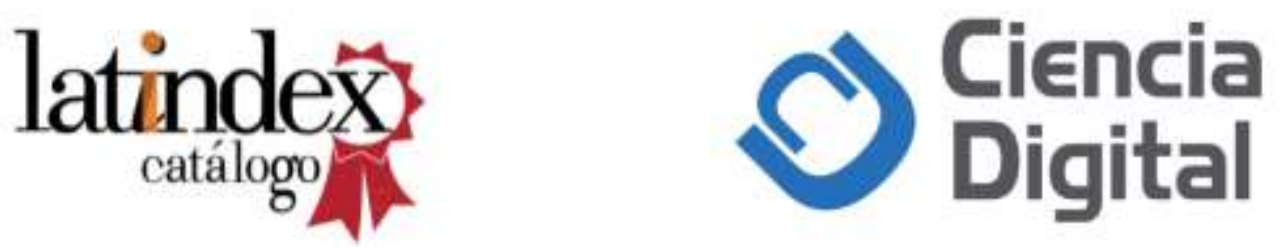